\title{
Acute Effects of Respiratory-Gated Auricular Vagal Afferent Nerve Stimulation (RAVANS) in the Modulation of Blood Pressure in Hypertensive Patients
}

\author{
Harris Fisher ${ }^{1}$, Jessica Stowell ${ }^{1,2}$, Ronald Garcia ${ }^{1,2}$, Roberta Sclocco ${ }^{1,3}$, \\ Jill Goldstein ${ }^{1,2}$, Vitaly Napadow ${ }^{1,3}$, Riccardo Barbieri ${ }^{4,5}$ \\ ${ }^{1}$ Athinoula A. Martinos Center for Biomedical Imaging, Department of Radiology, Massachusetts \\ General Hospital, Harvard Medical School, Boston, USA \\ ${ }^{2}$ Department of Psychiatry, Massachusetts General Hospital, Harvard Medical School, Boston, USA \\ ${ }^{3}$ Department of Radiology, Logan University, Chesterfield, USA \\ ${ }^{4}$ Department of Anesthesia, Critical Care and Pain Medicine, Massachusetts General Hospital, \\ Harvard Medical School, Boston, USA \\ ${ }^{5}$ Department of Electronics, Information and Bioengineering, Politecnico di Milano, Milano, Italy
}

\begin{abstract}
In this study, we evaluate the acute effects of a novel, non-invasive, respiratory-gated auricular vagal afferent nerve stimulation (RAVANS) technique on arterial blood pressure levels in hypertensive patients.

We consider data from 18 hypertensive subjects

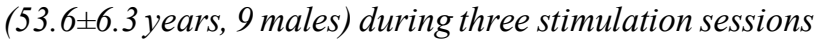
where they received either sham, low-intensity, or mediumintensity stimulation in randomized order. Blood pressure was continuously collected during 15-minute baseline, stimulation, and recovery windows.

Our statistical analysis shows that the percent decrease of median systolic blood pressure from baseline was significantly higher during RAVANS (medium-intensity) when compared to sham. Furthermore, a two-way ANOVA reveals a significant interaction between Intervention and Phase for changes in median systolic blood pressure. Post hoc testing reveals that this effect was driven by the Recovery Phase following RAVANS where median Systolic blood pressure significantly decreased in the last 5 minutes compared to sham.

Our results indicate that exhalatory-gated RAVANS has acute modulatory effects on systolic blood pressure levels of hypertensive patients. Further longitudinal studies will be required to evaluate the therapeutic potential of the RAVANS electroceutical device.
\end{abstract}

\section{Introduction}

Hypertension impacts $30 \%$ of the world's population and is a leading cause of morbidity and mortality in the United States [1]. It has been estimated that hypertension is responsible for $14 \%$ of deaths worldwide [2]. Though many medications for the condition exist, they are not effective for all individuals. In fact, half of all patients on hypertension medication still do not have adequately controlled blood pressure [3]. Furthermore, the worldwide population with uncontrolled hypertension increased from 605 million to 978 million between 1980 and 2008 [4]. This large population of hypertensive patients requires more effective treatment options, thus calling for exploration of alternative antihypertensive therapies.

Vagus Nerve Stimulation (VNS) is an FDA-approved treatment for treatment-resistant epilepsy and major depressive disorder [5,6]. VNS involves the surgical implantation of both a neurostimulator device and the electrode delivering the stimulation, which is placed along the cervical branch of the vagus nerve [7,8]. Clinical trials evaluating the potential of VNS as a therapeutic option for patients with heart failure have found that VNS is able to improve cardiac structure and function as well as increase parasympathetic tone $[9,10]$. Despite these promising results, the viability of VNS as a major treatment approach for other cardiovascular diseases, such as hypertension, is undercut by the risks associated with surgical implantation.

Non-invasive vagus nerve stimulation techniques have recently been developed to overcome the negative side effects involved when surgically implanting a medical device $[11,12]$. The cymba conchae, a central region of the outer ear, is highly innervated by the afferent pathway known as the auricular branch of the vagus nerve (ABVN), making it an ideal location for transcutaneous stimulation $[13,14]$. ABVN stimulation has been found to reduce arterial blood pressure and increase parasympathetic tone in preliminary experimental studies [15]. The link between reduced cardiac vagal tone and the development and progression of hypertension has been supported by the findings of many research groups [16-19]. Therefore, 
transcutaneous vagus nerve stimulation could provide a therapeutic tool for cardiovascular disorders associated with autonomic imbalance.

Our group has previously investigated a novel form of stimulation that is gated to the exhalatory phase of the respiration cycle $[20,21]$. We proposed that delivering stimulation during exhalation would optimize the effects of the stimulation in cardiovagal modulation [22]. In this study, we evaluate the effects of respiratory-gated auricular vagal afferent nerve stimulation (RAVANS) in the modulation of blood pressure in hypertensive subjects. We analyze the systolic blood pressure values of subjects randomized into active (low and medium intensity) or sham conditions by comparing the percent change from baseline blood pressure values during stimulation and recovery periods.

\section{Materials and methods}

\subsection{Experimental protocol}

Eighteen hypertensive subjects $(53.6 \pm 6.3$ years, 9 males) were enrolled in this study. Prior to enrollment in the study, all subjects were on stable doses of antihypertensive medications for a minimum of 30 days. Exclusion criteria included a history of other known cardio-, cerebro-, or peripheral vascular disease, presence of neoplasm, diabetes mellitus, morbid obesity, kidney or liver failure, infectious or systemic inflammatory disease and current neurological illness. All subjects gave written informed consent approved by an Institutional Review Board. Participants underwent three stimulation sessions, during which they received either sham, low-intensity, or medium-intensity stimulation in a randomized order (see below). Electrodes were placed on the left ear, specifically on the surface of the cymba concha and beneath the antihelix as these regions are known to be innervated by the auricular branch of the vagus nerve (Figure 1). Biphasic electrical stimulation was delivered at $25 \mathrm{~Hz}$ (duration 1s, pulse width 15ms) and was gated to exhalation, with current intensity set to achieve a non-painful, mild or moderate sensation. Subjects rested in a seated position for non-consecutive 15-minute baseline, stimulation, and recovery periods.

Figure 1. Electrode placement in the cymba concha

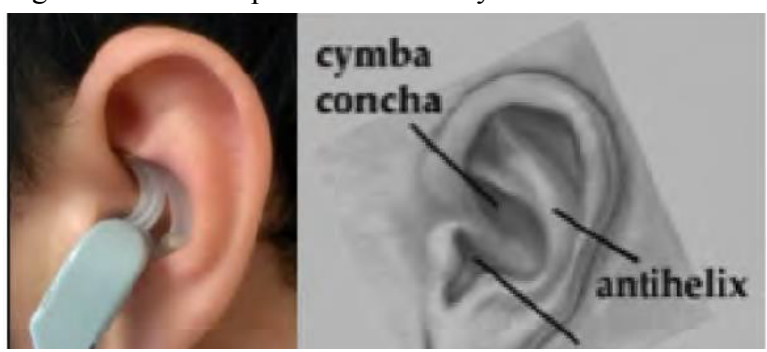

Stimulation intensity was determined by a subjective rating scale of $0-10$ for perceived intensity of the sensation. On the scale, an intensity rating of ' 0 ' represented no sensation and a rating of ' 10 ' represented a sensation approaching pain threshold. Low-intensity stimulation corresponded to a subjective rating of ' 2 ' on the scale, and medium-intensity stimulation to a rating of ' 5 '. The sham stimulation was delivered with the current generator shut off, with subjects blinded to stimulus condition. For the sham condition, participants were told they may not feel the stimulation because the intensity adopted could be below sensory-threshold. Continuous beat-to-beat blood pressure signal was measured by a Finometer device (Finapres Medical System, Enschede, The Netherlands) throughout each session. The signal was collected at $400 \mathrm{~Hz}$ using a 16-channel Powerlab DAQ System (ADInstruments, Colorado Springs, CO, USA) on a laptop equipped with LabChart Data Acquisition Software (ADInstruments).

\subsection{Statistical Analysis}

Systolic peaks of the blood pressure data were annotated semi-automatically_peaks were automatically detected and then manually inspected and corrected if needed - with in-house developed Matlab scripts (The MathWorks, Natick, MA, USA). The annotated data were segmented into five-minute windows in order to visually inspect and exclude any low-quality segments. The median systolic blood pressure value was extracted for each of the selected windows. Statistical testing was performed using R (http://www.R-project.org/). A first analysis investigated blood pressure variations during stimulation. To this end, the median percent signal change in systolic BP (SBP) during stimulation was computed using the baseline period as a reference. RAVANS was compared to Sham using a Wilcoxon paired test, and significance was set at a p-value of 0.05. Additionally, the subjects included in this pairwise analysis were inspected in order to identify potential responders and non-responders. Finally, the overall effect of RAVANS was assessed by evaluating the median percent signal change in SBP in the last 5 minutes of each period (baseline, stimulation, recovery) with respect to the first 5 minutes. A two-way ANOVA with Period (three levels: baseline, stimulation, recovery) and Stimulation Type (three levels: Low Intensity, Medium Intensity, Sham) as factors was performed, followed by post-hoc Wilcoxon testing.

\section{Results}

Through a preliminary preprocessing of the data based on quality, ten subjects $(\mathrm{N}=10)$ were retained for analyses comparing median systolic blood pressure values between active and sham RAVANS during the stimulation period. Nine out of these ten subjects showed a lower increase in SBP during active medium intensity RAVANS compared to sham (Figure 2). For the responder group, SBP change was significantly lower during medium intensity 
RAVANS $(0.86+/-8.34 \%$ mean $+/-$ sd) than sham stimulation $(7.94+/-7.06 \% ; \mathrm{p}=0.04)$. The non-responder had a SBP change of $16.34 \%$ during medium intensity stimulation and $-2.01 \%$ during sham.

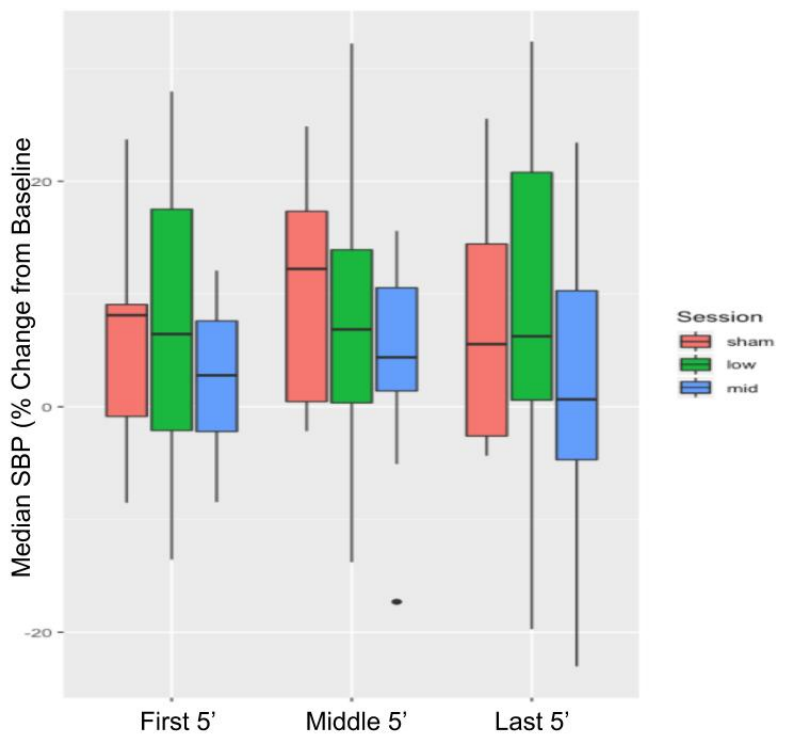

Figure 2. Median systolic blood pressure, normalized to baseline, is lower during stimulation for medium intensity RAVANS than sham for nine out of ten subjects (all ten subjects' data shown).

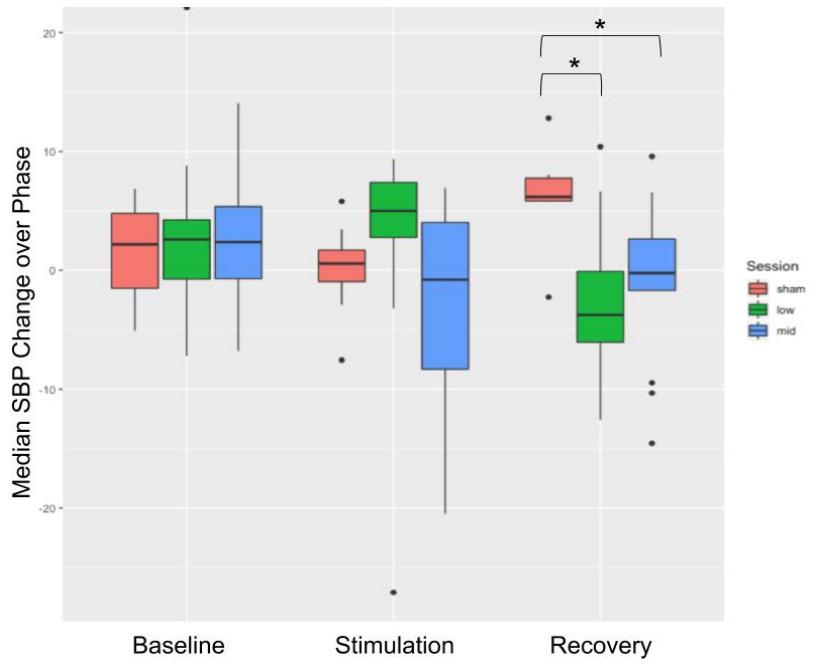

Figure 3. Median systolic blood pressure of the final five minutes of each phase expressed as a percent change from the first five minutes. Both low and medium intensity stimulation have significantly greater blood pressure reductions compared to sham $(* \mathrm{p}<0.05)$.

The $3 \times 3$ ANOVA on the percent change SBP values revealed no significant main effect of Phase or Stimulation Type, but a significant interaction between factors $(\mathrm{F}(4)=$ 2.748, $\mathrm{p}=0.03$ ). Post-hoc analyses revealed that SBP changes were higher during recovery following sham stimulation $(6.28+/-4.16 \%$, mean $+/-$ std $)$ and lower following both medium $(-0.80+/-6.75 \%)$ and low $(-2.49$
$+/-7.03 \%$ ) intensity stimulation (Figure 3). Wilcoxon rank sum testing demonstrates significance differences between sham and low RAVANS ( $\mathrm{p}=0.027)$, and between sham and medium RAVANS $(\mathrm{p}=0.024)$.

\section{Discussion}

The present study investigates the acute effects of exhalatory-gated transcutaneous VNS (RAVANS) on systolic blood pressure in hypertensive subjects. Specifically, we explored the influence of stimulus intensity as a potentially relevant parameter for clinical applications. Our findings show a decrease in normalized systolic BP during the Medium intensity RAVANS stimulation period compared to sham stimulation for a subgroup ( 9 out of 10 subjects) of responders. Furthermore, subjects who received active stimulation (either using medium or low intensity) showed a significantly lower SBP increase at the end of the recovery period compared to sham.

Pooled together, the present results provide preliminary support for antihypertensive effects of RAVANS stimulation in our hypertensive population. Importantly, both medium and low intensity showed a significant effect in maintaining a controlled SBP during a 15-minute recovery period compared to sham (no current) stimulation. Combining SBP analysis with other autonomic and cardiovascular indices, such as the ones derived from heart rate variability analysis, could offer a more comprehensive picture of the effects of RAVANS and its stimulation intensity - on hypertension, thus helping an informed decision on this parameter.

The study has a number of limitations that should be discussed. First, the number of subjects included in the analysis is reduced, and could be increased by further assessments on data quality. A second, important limitation is given by the protocol design: in fact, the subjects underwent a series of autonomic tasks (paced breathing, hand grip, sit-to-stand) preceding and following the 15-minute stimulation period, whose effects on BP levels have not been yet ascertained. A new protocol where such effect is minimized could further improve validation of the RAVANS effect.

\section{Conclusion}

The preliminary findings of this study provide promising evidence that RAVANS holds the potential to regulate arterial blood pressure levels of patients with hypertension. Future studies targeting the mechanism by which RAVANS influences parasympathetic activity and thereby, modulates blood pressure, would be helpful in determining the therapeutic value of this technique. As more data on use of RAVANS in hypertensive subjects become available, the ability of this novel form of 
stimulation to reduce cardiovascular risk can be further validated.

\section{Acknowledgements}

This study was supported by the Department of Anesthesia, Critical Care and Pain Medicine at Massachusetts General Hospital; the American Heart Association (Grant No. 16GRNT26420084), NIH-Office of Director (OT2-OD023867), the European Commission LINK project (H2020-692023) and by the Boston Biomedical Innovation Center (B-BIC), a National Center for Accelerated Innovation funded by the National Heart, Lung, and Blood Institute of the National Institutes of Health under Award Number U54HL119145.

\section{References}

[1] Ezzati M, Oza S, Danaei G, Murray CJL. Trends and cardiovascular mortality effects of state-level blood pressure and uncontrolled hypertension in the United States. Circulation. 2008;117(7):905-14.

[2] Ezzati M, Hoorn SV, Lopez AD, Danaei G, Rodgers A, Mathers CD, et al. Comparative Quantification of Mortality and Burden of Disease Attributable to Selected Risk Factors. In: Lopez AD, Mathers CD, Ezzati M, Jamison DT, Murray CJL, editors. Global Burden of Disease and Risk Factors. Washington (DC): World Bank The International Bank for Reconstruction and Development/The World Bank Group.; 2006.

[3] Kearney PM, Whelton M, Reynolds K, Muntner P, Whelton PK, He J. Global burden of hypertension: analysis of worldwide data. Lancet. 2005;365(9455):217-23.

[4] Danaei G, Finucane MM, Lin JK, Singh GM, Paciorek CJ, Cowan MJ, et al. National, regional, and global trends in systolic blood pressure since 1980: systematic analysis of health examination surveys and epidemiological studies with 786 country-years and 5.4 million participants. Lancet. 2011;377(9765):568-77.

[5] Daban C, Martinez-Aran A, Cruz N, Vieta E. Safety and efficacy of vagus nerve stimulation in treatment-resistant depression. A systematic review. J Affect Disord. 2008;110(1-2):1-15.

[6] Englot DJ, Chang EF, Auguste KI. Vagus nerve stimulation for epilepsy: a meta-analysis of efficacy and predictors of response. J Neurosurg. 2011;115(6):1248-55.

[7] George MS, Nahas Z, Bohning DE, Lomarev M, Denslow S, Osenbach R, et al. Vagus nerve stimulation: a new form of therapeutic brain stimulation. CNS Spectr. 2000;5(11):4352.

[8] Nemeroff CB, Mayberg HS, Krahl SE, McNamara J, Frazer A, Henry TR, et al. VNS therapy in treatment-resistant depression: clinical evidence and putative neurobiological mechanisms. Neuropsychopharmacology. 2006;31(7):134555.

[9] De Ferrari GM, Schwartz PJ. Vagus nerve stimulation: from pre-clinical to clinical application: challenges and future directions. Heart Fail Rev. 2011;16(2):195-203.

[10] De Ferrari GM, Crijns HJGM, Borggrefe M, Milasinovic G, Smid J, Zabel M, et al. Chronic vagus nerve stimulation: a new and promising therapeutic approach for chronic heart failure. Eur Heart J. 2011;32(7):847-55.

[11] Ventureyra EC. Transcutaneous vagus nerve stimulation for partial onset seizure therapy. A new concept. Childs Nerv Syst. 2000;16(2):101-2.

[12]Ellrich J. Transcutaneous vagus nerve stimulation. Eur Neurol Rev. 2011;6(4):254-6.

[13] Peuker ET, Filler TJ. The nerve supply of the human auricle. Clin Anat. 2002;15(1):35-7.

[14] Bermejo P, Lopez M, Larraya I, Chamorro J, Cobo JL, Ordonez S, et al. Innervation of the human cavum conchae and Auditory Canal: Anatomical Basis for Transcutaneous Auricular Nerve Stimulation. 2017;2017:7830919.

[15] Gao XY, Li YH, Liu K, Rong PJ, Ben H, Li L, et al. Acupuncture-like stimulation at auricular point heart evokes cardiovascular inhibition via activating the cardiac-related neurons in the nucleus tractus solitarius. Brain Res. 2011;1397:19-27.

[16] Grossman P, Brinkman A, de Vries J. Cardiac autonomic mechanisms associated with borderline hypertension under varying behavioral demands: evidence for attenuated parasympathetic tone but not for enhanced beta-adrenergic activity. Psychophysiology. 1992;29(6):698-711.

[17] Radaelli A, Bernardi L, Valle F, Leuzzi S, Salvucci F, Pedrotti L, et al. Cardiovascular autonomic modulation in essential hypertension. Effect of tilting. Hypertension. 1994;24(5):556-63.

[18] Mancia G, Grassi G. The autonomic nervous system and hypertension. Circ Res. 2014;114(11):1804-14.

[19] Thayer JF, Yamamoto SS, Brosschot JF. The relationship of autonomic imbalance, heart rate variability and cardiovascular disease risk factors. Int $\mathrm{J}$ Cardiol. 2010;141(2):122-31.

[20] Napadow V, Edwards RR, Cahalan CM, Mensing G, Greenbaum S, Valovska A, et al. Evoked pain analgesia in chronic pelvic pain patients using respiratory-gated auricular vagal afferent nerve stimulation. Pain Med. 2012;13(6):77789.

[21] Garcia RG, Lin RL, Lee J, Kim J, Barbieri R, Sclocco R, et al. Modulation of brainstem activity and connectivity by respiratory-gated auricular vagal afferent nerve stimulation in migraine patients. Pain. 2017;158(8):1461-72.

[22] Sclocco R, Garcia RG, Gabriel A, Kettner NW, Napadow V, Barbieri R. Respiratory-gated auricularvVagal afferent nerve stimulation (RAVANS) effects on autonomic outflow in hypertension. Conf Proc IEEE Eng Med Biol Soc. 2017;2017:3130-3.

Address for correspondence.

Riccardo Barbieri

Politecnico di Milano, Via Ponzio 34/5, 20133, Milano, Italy

Riccardo.barbieri@polimi.it 